\title{
Neurotoxicité du métronidazole
}

\author{
Danica Quickfall MD CM, Nick Daneman MD, Adam A. Dmytriw MD MSP, David N. Juurlink MD PhD
}

Citation : CMAJ 2021 October 25;193:E1630. doi : 10.1503/cmaj.201671-f

Voir la version anglaise de l'article ici : www.cmaj.ca/lookup/doi/10.1503/cmaj.201671

1 La neurotoxicité du métronidazole est sous-estimée

Habituellement prescrit pour des infections dues à des bactéries anaérobies et à des protozoaires, le métronidazole peut dans de rares cas causer des effets indésirables qui affectent le système nerveux central dont le mécanisme précis reste à élucider. Dans une étude cas-témoins, l'incidence des complications neurologiques a été de 0,25\%, mais il s'agit probablement d'une sous-estimation ${ }^{1}$.

\section{La neurotoxicité du métronidazole peut se manifester par divers syndromes cliniques}

Les caractéristiques fréquentes impliquant le système nerveux central incluent l'ataxie, la dysarthrie et l'altération de l'état mental, mais des manifestations plus rares comme des convulsions, l'encéphalopathie et la dysfonction cérébelleuse ont aussi été décrites ${ }^{2}$. La neuropathie périphérique prend la forme d'une diminution de la sensibilité, d'engourdissements et de douleur neuropathique. Environ le tiers des patients qui présentent des anomalies du système nerveux central manifesteront aussi des signes de neuropathie périphérique².

\section{3}

La dose et la durée du traitement sont des facteurs de risque

importants, mais les symptômes peuvent apparaître tôt, et même avec des petites doses

Les rapports de cas laissent entendre qu'il peut s'écouler en moyenne 6-7 semaines avant l'apparition des symptômes. Or, ces derniers peuvent se manifester en aussi peu que quelques jours après l'instauration du traitement $^{2,3}$. Les patients sous métronidazole pour une maladie inflammatoire de l'intestin, l'ostéomyélite ou de volumineux abcès non drainés sont exposés à un risque plus élevé de neurotoxicité en raison de la durée de l'exposition. Une revue de 110 cas d'encéphalopathie induite par le métronidazole chez des adultes a fait état d'une dose cumulative médiane de $65,4 \mathrm{~g}$, mais la variabilité était importante (éventail 5-2000 g)².

Jusqu'à $90 \%$ des patients qui ont une atteinte du système nerveux central présentent des lésions caractéristiques à l'imagerie par résonance magnétique (IRM)

Les signes radiologiques typiques s'observent le plus souvent à l'IRM pondérée en $T_{2}$, en séquence FLAIR (fluid-attenuated inversion recovery). Les anomalies souvent présentes sont des lésions symétriques hyperintenses au niveau des noyaux dentelés, avec parfois des lésions au niveau du splénium du corps calleux et de la partie dorsale du pont de Varole émettant les mêmes types de signaux caractéristiques ${ }^{2,4}$.

Le JAMC vous invite à soumettre vos textes pour la rubrique " Cinq choses à savoir ... » en ligne à http://mc.manuscriptcentral.com/cmaj.

\section{L'arrêt du métronidazole donne 3 généralement lieu à une amélioration} La plupart des patients obtiennent une résolution complète de leurs symptômes à l'arrêt du métronidazole et une résolution radiologique complète ou quasi complète s'observe dans environ $75 \%$ des $\operatorname{cas}^{2,4}$. L'évolution chronologique varie, mais les symptômes, chez la plupart des patients rentrent dans l'ordre en l'espace de 2 semaines ${ }^{4}$.

\section{Références}

1. Daneman N, Cheng Y, Gomes T, et al. Metronidazole-associated neurologic events: a nested case-control study. Clin Infect Dis 2021;72:2095-100

2. Sørensen CG, Karlsson WK, Amin FM, et al. Metronidazoleinduced encephalopathy: a systematic review. J Neurol 2020; 267:1-13.

3. Kuriyama A, Jackson JL, Doi A, et al. Metronidazole-induced central nervous system toxicity: a systematic review. Clin Neuropharmacol 2011;34:241-7.

4. Patel L, Batchala P, Almardawi R, et al. Acute metronidazoleinduced neurotoxicity: an update on MRI findings. Clin Radiol 2020;75:202-8.

Intérêts concurrents : Aucun déclaré.

Cet article a été révisé par des pairs.

Affiliations : Département de médecine (Quickfall, Daneman, Juurlink), Centre des sciences de la santé Sunnybrook; Départements de médecine et de pédiatrie (Quickfall, Daneman, Juurlink), Université de Toronto; Institut de recherche en services de santé (ICES) (Daneman, Juurlink), Toronto, Ont.; Programme neuroendovasculaire (Dmytriw), Hôpital général du Massachusetts, Faculté de médecine de l'Université Harvard, Boston, Mass.; Centre antipoison de l'Ontario (Juurlink), Toronto, Ont.

Propriété intellectuelle du contenu : Il s'agit d'un article en libre accès distribué conformément aux modalités de la licence Creative Commons Attribution (CC BY-NC-ND 4.0), qui permet l'utilisation, la diffusion et la reproduction dans tout médium à la condition que la publication originale soit adéquatement citée, que l'utilisation se fasse à des fins non commerciales (c.-à-d., recherche ou éducation) et qu'aucune modification ni adaptation n'y soit apportée. Voir : https:// creativecommons.org/licenses/by-nc-nd/4.0/deed.fr.

Correspondance : David Juurlink, david.juurlink@ices.on.ca 